\title{
Construção e Compreensão de Saberes com Experiências Didáticas com Objetos de Aprendizagem desde a Formação Inicial de Professores de Matemática
}

\author{
Raquel G. Oliveira ${ }^{1}$, Elvis. M. Barbosa ${ }^{1}$, Juliane Santos $^{1}$, Mara Zanini ${ }^{1}$ \\ ${ }^{1}$ Faculdade de Ciências e Tecnologia - FCT - Universidade Estadual Paulista (UNESP) \\ Presidente Prudente - SP - Brazil \\ raqueloliveiradfct.unesp.br, elvis marciodig.com.br, \\ ju jullyane@hotmail.com, marazanini2@hotmail.com
}

\begin{abstract}
In this paper we describe didactic experiences with learning object (LO) in mathematics classroom regular situations. The purpose of these experiments was not to examine clinically the $L O$ and its features, but considering their use in regular classroom settings in order to elucidate knowledge and implications for the process of teaching and learning for both the student of basic education as to future mathematics teachers who will work at this level of teaching. The didactic experiences resulted in the development of parameters to future teachers for the systematization of a mathematical formula using a LO.
\end{abstract}

Resumo. Descrevemos neste artigo experiências didáticas com objeto de aprendizagem (OA) em situações regulares de aula de Matemática. O objetivo dessas experiências não era o de analisar clinicamente o OA e suas características, mas sim de verificarmos a utilização dos mesmos em contextos regulares de sala de aula tendo em vista elucidar saberes e implicações para o processo de ensino e aprendizagem tanto para o aluno da Educação Básica como para os futuros professores de Matemática que atuarão neste nível de ensino. As experiências didáticas resultaram na elaboração de parâmetros, aos futuros professores, para a sistematização de uma fórmula matemática utilizando-se um $O A$.

\section{Introdução}

A utilização de informática na educação brasileira teve início nos anos 70 e, mesmo com dificuldades e em alguns casos fracassos, tem insistido no objetivo de defender o entendimento de que aportes tecnológicos devem contribuir para efetivas ações de construção, de reelaboração e de uso de conhecimento. Nesse sentido, torna-se imprescindível oportunizar referências de utilização de informática no processo de ensino e aprendizagem, desde a formação inicial de professores. Respondendo às demandas sociais decorrentes fundamentalmente das consequências da utilização da informática na educação e encontrando respaldo em políticas públicas, países latinos americanos como Peru, Chile, Paraguai, Uruguai, Argentina, Colômbia e Brasil organizaram coleções online de recursos educacionais digitais, como por exemplo, objetos de aprendizagem (OAs) e objetos educacionais (OEs), que se encontram disponíveis em repositórios digitais (COLOMBIA APRENDE, 2004; BIOE, 2008). 
De acordo com Fabri et. al. (2012) existem vários exemplos de bem sucedidos ROAs (Repositórios de Objetos de Aprendizagem). Os repositórios de objetos de aprendizagem, por sua vez, tiveram origem em uma das ideias que permeiam a sociedade atual: a ideia de inteligência coletiva (LEVY, 1993, 1998) com seu constante crescimento que encontra incentivo e suporte em acervos, de natureza cada vez mais virtual ou digital, de materiais informativos e educacionais. O conceito de inteligência coletiva possui como pilar fundamental os saberes tecnológicos.

Para a International Society for Technology in Education (ISTE, 2009), saberes tecnológicos, que incluem a utilização de tecnologias de informação e comunicação, inclusive em educação, levam professores e alunos a: desenvolver conhecimentos, habilidades e disposições pessoais (atitudes) quanto a estratégias de aprendizagem, formulando perguntas significativas sobre um tema, podendo alcançar e selecionar fontes de adequada informação que respondam a essas perguntas e adquirir habilidades que lhe permitam aprender de modo autônomo e colaborativo por toda a vida.

Entendendo que utilizar informática na educação deve ser associado a efetivos saberes docentes e atentando igualmente para as positivas consequências deste uso em processos pedagógicos é que utilizamos 3 diferentes objetos de aprendizagem (OAs) em aulas regulares de Matemática da Educação Básica. O objetivo desta utilização não era analisar cada OA e suas características fazendo relações e associações entre a aprendizagem e o desenvolvimento do pensamento algébrico. Este tipo de análise sobre utilização de OA para colaborar no desenvolvimento especificamente de representações e pensamentos algébricos de alunos da Educação Básica foi realizado por Macedo et.al. (2008).

Nosso objetivo, ao realizar atividades didáticas com OAs, era elucidar saberes e implicações para o processo de ensino e aprendizagem tanto para o aluno da Educação Básica como para os futuros professores de Matemática que atuarão neste nível de ensino. Defendemos que essas implicações têm origem tanto na utilização de OAs como na consideração dessa realização em contextos regulares de sala de aula.

\section{Sobre objetos de aprendizagem para o processo de ensino e aprendizagem}

Os parâmetros curriculares nacionais brasileiros (PCNs, 1998) apontam para a importância da utilização da informática no processo de ensino e aprendizagem de Matemática na medida em que esta utilização seja colaboradora de atividades didáticas nas quais os alunos tenham oportunidades de serem efetivos participantes e o professor experiencie o papel que pode assumir na mediação entre conhecimentos prévios dos alunos e os conceitos matemáticos curriculares. Essa mediação (MASETTO, 2000) deve permitir que o aluno reflita sobre sua ação e pensamentos, reelaborando outras ações para realização de atividades e alcance de seus objetivos.

Há propostas e pesquisas sobre a utilização de informática na educação (LEITE, CASTRO, 2006; PRATA, 2007; DIRENE et. al., 2009; BARBOSA et. al, 2010) que resultaram no entendimento de que objetos de aprendizagem possuem potencialidades para o processo de mediação didática. Essas potencialidades coadunam com a definição de objeto de aprendizagem considerada por nós nas experiências didáticas.

Um objeto virtual de aprendizagem é um recurso digital reutilizável que auxilie na aprendizagem de algum conceito e, ao mesmo tempo, e estimule o desenvolvimento de capacidades pessoais, como por exemplo, imaginação e 
criatividade. Dessa forma, um objeto virtual de aprendizagem pode contemplar um único conceito quanto englobar todo o corpo de uma teoria. Pode ainda compor um percurso didático, envolvendo um conjunto de atividades, focalizando determinado aspecto do conteúdo envolvido, ou formando com exclusividade a metodologia adotada para determinado trabalho. (SPINELLI, 2007 apud AUDINO E NASCIMENTO, 2010, p. 131).

Assim, infere-se que um OA é um elemento intrínseco à utilização de informática na educação porque apresenta potencialidades para contribuir com o processo pedagógico em situações de ensino aprendizagem. É necessário evitar a distorção, ainda realizada, que considera que um $\mathrm{OA}$, por si, já é um recurso pedagógico. Se assim fosse, a profissão docente, com seus saberes e procedimentos específicos seria dispensável. Fato desconsiderado quando se utilizam TICs (Tecnologias de Informação e Comunicação) diante de princípios que explicitam o papel das mesmas na prática docente. (VALENTE, 1999, MASETTO, 2000, KENSKI, 2003). Nesse artigo descreveremos a utilização de 3 objetos de aprendizagem, em situações didáticas de aulas de Matemática, salientando suas efetivas contribuições aos processos formativos para a aprendizagem de Matemática dos alunos e para o estabelecimento de referências de ensino para futuros professores de Matemática.

\section{O Trabalho Pedagógico com Objetos Educacionais desde a formação inicial de professores de Matemática}

\subsection{Sobre a experiência com o Tangran Digital}

A experiência didática com o objeto de aprendizagem Tangran ocorreu com 38 alunos de uma $8^{a}$ série ( $9^{\circ}$ ano), de uma escola estadual pública do interior de São Paulo e foi realizada no laboratório de informática da escola com a utilização de 20 computadores que possuíam acesso à internet. A aula tinha como objetivo levar os alunos a construir o conceito de figuras geométricas planas por meio de utilização de uma simulação digital. Esta simulação utilizou-se do quebra cabeça chinês denominado Tangran. O Tangran digital utilizado nesta experiência foi produzido pela Estação Ciência do Centro de Difusão Científica, Tecnológica e Cultural da Pró-Reitoria de Cultura e Extensão Universitária da Universidade de São Paulo. Este Tangran é também um tangran de sete peças com colorações distintas e seis modelos de figuras que podem ser construídas com a junção das peças disponíveis, permitindo levar o aluno a perceber e a refletir sobre as diversas formas geométricas que podem ser geradas a partir de uma figura inicial, através de sua composição e decomposição. Primeiramente, o Tangran foi apresentando aos alunos, que receberam explicações sobre as formas de rotacionar e deslocar as peças da simulação (figura 1).

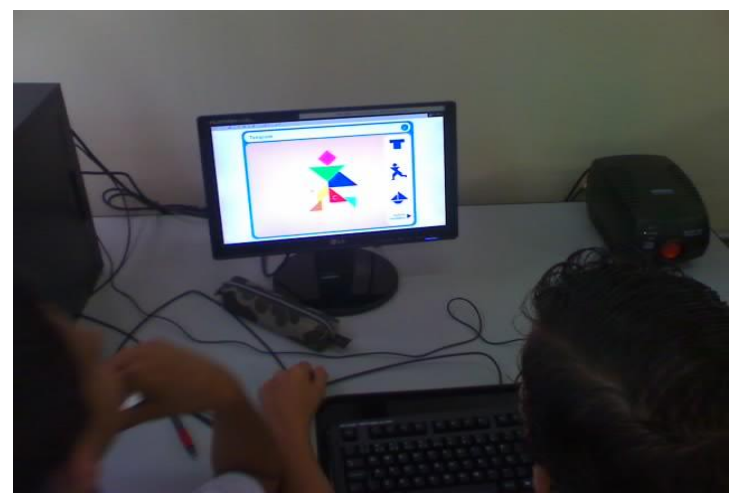


Figura 1 - Alunos utilizando as peças do OE Tangran

Logo após, os alunos deveriam preencher a tabela 1, registrando o número de peças utilizadas na construção de cada figura.

\section{Tabela 1 - Composição de figuras geométricas com as peças do Tangran}

\begin{tabular}{|l|l|l|l|l|l|l|l|}
\hline \multicolumn{1}{|c}{ No dep pecas $^{\text {Trängulos }}$} & 1 & 2 & 3 & 4 & 5 & 6 & 7 \\
\hline Quadrados & $\square$ & & & & & & \\
\hline Losangos & & & & & & & \\
\hline Retángulos & & & $\square$ & & & & \\
\hline Trapezzios & & & & & & & \\
\hline Paralelogramos & & & & & & & \\
\hline
\end{tabular}

Para preencherem a tabela, os alunos foram orientados a observar as informações da mesma. Exemplo disto foi a construção de um retângulo utilizando apenas uma peça do Tangran até atingir o uso das sete peças disponíveis. Quando não era possível essa construção com o número de peças solicitadas, o aluno poderia registrar essa ocorrência preenchendo o interior do mesmo campo com as palavras "não compõem". Após a confecção da tabela, iniciamos a discussão dos resultados com os alunos sobre a composição e a decomposição geométrica das várias figuras ali presentes, com a finalidade de verificar se conseguiram identificar as formas geométricas envolvidas e a exploração dos conceitos de área e de perímetro das mesmas.

\subsection{Sobre a experiência com a Sequência de Fibonacci ou com o Problema dos Coelhos}

Essa experiência didática foi realizada com 3 classes de $8^{a}$ série $\left(9^{\circ}\right.$ ano), tendo 38 alunos cada, de uma escola estadual pública do interior de São Paulo, tendo acontecido no laboratório de informática da escola, onde ocorreram as aulas de Matemática que utilizaram o OA. Em todos os computadores era possível acessar a internet.

Iniciamos nossa atividade didática com a apresentação do problema denominado de "problema dos coelhos": qual o número de pares de coelhos que serão gerados num ano a partir de um casal de coelhos jovens, nas seguintes condições: 1) nenhum coelho morre durante o ano; 2) cada casal de coelhos gera, a cada mês, outro casal de coelhos e 3) cada coelho (fêmea) fica fértil após dois meses?

Para resolver o problema foi utilizado o OA O Problema dos Coelhos- Leonardo de Pisa (figura 2), que é uma simulação digital produzida pela Ludoteca do Instituto de Física da USP. Ao utilizar o OA, percebe-se que existem 2 tipos de casais de coelhos: um casal jovem representado por 2 coelhos de menor estatura e um casal adulto formado por 2 coelhos maiores. A simulação digital possui um quadro que informa $o$ número de casais a cada vez que o aluno avançar sobre o período de 12 meses e o mês selecionado, sendo este último de forma sequencial com início em janeiro e término no 
mês de dezembro. Em uma tela maior ao lado surgem coelhos grandes e pequenos que totalizam os casais em um determinado mês.

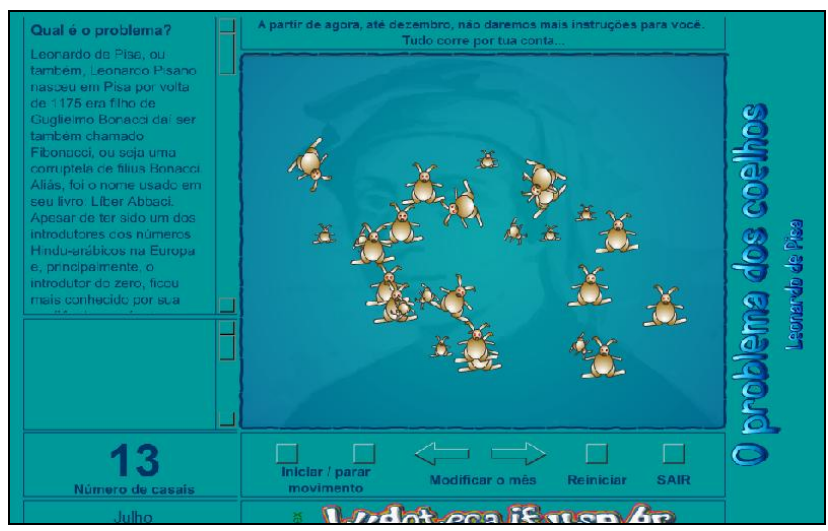

Figura 2: Tela do AO o Problema dos coelhos - Leonardo de Pisa

Fonte:http://www.cienciamao.usp.br/dados/tex/_fibonacciproblemadoscoelhos.flash.swf

Como parte de resolução do problema, os alunos deveriam utilizar o OA para preencher uma tabela dividida em 4 colunas e que tinha por finalidade disponibilizar informações, tais como: meses do ano, casal de coelhos jovens, casal de coelhos adultos e número total de casais. Para o preenchimento da tabela, os alunos foram orientados a levantar dados de acordo com o que fosse observado na utilização do OA.

A disponibilização dos dados na tabela permitiu que os alunos fossem orientados a refletirem sobre possíveis regularidades dos mesmos. Ou seja, levávamos os alunos a perceberem se havia algo que se repetia. Isto se deu através de questões propostas para a reflexão dos alunos. Uma destas questões enfatizava o fato de haver ou não uma dependência dos números de uma linha da tabela com os da linha anterior.

Os resultados da atividade didática foram satisfatórios, pois a maior parte dos alunos conseguiu perceber que a soma entre o número de casais jovens e adultos gera o número de casais adultos do seguinte mês e que o número de casais adultos de um mês gera o total de casais jovens no mês seguinte. Portanto, o encontro de regularidades dos dados permitiu a formalização do conceito de sequências numéricas, precisamente a Sequência de Fibonacci.

\subsection{Sobre a experiência com a Fórmula e Pick}

A experiência didática sobre a Fórmula de Pick foi desenvolvida com 10 alunos de uma turma de $8^{\circ}$ ano de uma escola estadual pública do interior de São Paulo. A Fórmula de Pick permite calcular a área de um polígono simples a partir da contagem de pontos do reticulado no qual este polígono está inscrito. Nosso objetivo era levar os alunos à sistematização da Fórmula de Pick por meio da participação nas aulas reelaboradas a partir da aula "Área de Figuras Poligonais em Malha Pontilhada". Todas as aulas aconteceram na sala de informática da escola $\mathrm{cm}$ computadores que possuíam acesso à internet.

Com o objetivo de saber sobre os conhecimentos e dificuldades dos alunos quanto aos conceitos de área de figuras geométricas planas em uma malha quadriculada, 
pois este tipo de contexto de cálculo de área já é conhecido dos alunos, aplicamos um pré-teste. Os alunos puderam escolher dentre três métodos para o calculo de área das figuras, esses métodos eram: Método de cálculo de área através de um Exemplo Dado; Método de cálculo de área através de Contagem e Método de cálculo de área através de Aproximação.

De maneira geral, os resultados encontrados no pré-teste confirmaram nossa hipótese na qual apresentar aos alunos a Fórmula de Pick, no modo sistematizado, seguido de um exemplo de uma aplicação prática para depois pedirmos exercícios semelhantes ao exemplo dado, certamente, para esses alunos não seria proveitoso didaticamente. Isto porque os resultados do pré-teste apontaram dificuldades primárias ligadas: 1) ao cálculo da área de figuras geométricas conhecidas, tais como: retângulo, quadrado, triângulo; 2) à utilização da contagem de uma dada unidade de medida para se chegar à área de uma região e 3) à interpretação de um exemplo dado para o cálculo de área por aproximação. Amparadas por esses resultados, pesquisamos materiais didáticos e sugestões de aula de Matemática que estão hospedados no Portal do Professor do MEC e que revelassem princípio de atividades de investigação matemática. (PONTE et. al., 1999).

O trabalho didático com a Fórmula de Pick buscou desenvolver as atividades de cálculo de áreas de figuras geométricas (retângulo, triângulo, trapézio...) inicialmente com fórmulas conhecidas dos alunos. Estes também deveriam preencher uma tabela com características de cada figura: número de lados, número de pontos internos da figura (I) e número de pontos pelos quais passam as poligonais da figura (B).

A fim de responder às questões: existe alguma relação entre o valor encontrado para a área da figura e os valores de I e de B? Se existe, é possível expressar essa relação na forma de uma fórmula matemática? Como seria essa fórmula? Os alunos utilizaram dados da tabela, mas também construíram e realizaram os mesmos procedimentos com várias figuras que puderam construir utilizando o OA da figura 3.

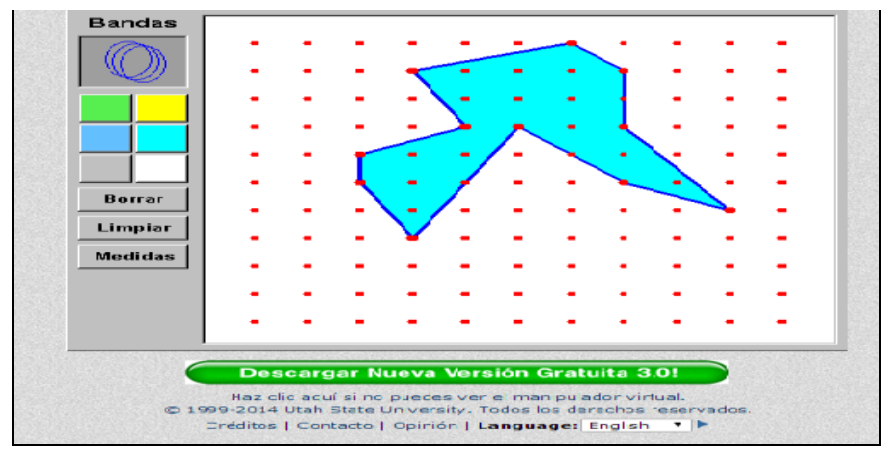

Figura: Tela do OA Geoplano virtual

Fonte: http://nlvm.usu.edu/es/nav/frames asid $277 \mathrm{~g} 1 \mathrm{t}$ 3.html

\section{Resultados e discussões das experiências didáticas}

De maneira geral, os resultados das 3 experiências permitem reconhecer que um objeto de aprendizagem (OA) é elemento positivamente contribuinte no processo de ensino e aprendizagem que atente para condições e necessidades dos alunos na construção e domínio cognitivo de conteúdos curriculares. 
No trabalho com o Tangran digital foi possível aos alunos perceberem que uma figura geométrica pode ser composta e decomposta em outras figuras geométricas, o que permitiu ampliar as ideias referentes aos conceitos de área e de perímetro de uma figura. $\mathrm{O}$ fato de os alunos serem responsáveis pela conduta do processo de experimentação, tendo uma sequência de atividades para desenvolver, contribuiu para que entendêssemos a necessidade de planejamento para se utilizar um OA. A formação de pares para utilização de tecnologia ocasionou reflexões conjuntas e este ato se intensificou, gerando um debate entre todos, no momento em que os alunos buscavam encontrar meios de junção das figuras geométricas para o preenchimento de cada um dos campos da tabela. Kenski (2007) chama a atenção para o desafio de criar planos de aula ou situações de ensino e aprendizagem com o uso de tecnologia e que possam modificar o ambiente de aprendizagem gerando a participação ativa e a colaboração de todos.

O desafio é o de inventar e descobrir usos criativos da tecnologia educacional que inspirem professores e alunos a gostar de aprender, para sempre. A proposta é ampliar o sentido de educar e reinventar a função da escola, abrindo-a para novos projetos e oportunidade. (KENSKI, 2007, p.67).

Nesta nossa experiência de utilizar OA desde a formação inicial de professores, foi possível constatar que essa utilização oportuniza ao aprendiz a criação de múltiplas realidades, por meio de simulações com diferentes resultados, que auxiliam o professor no processo de ensino e aprendizagem. Vivenciamos esse auxílio quando sentimos os alunos estimulados a participarem de seu processo de aprendizagem, de forma ativa e com significado para os mesmos. A partir das experimentações realizadas pelos alunos, pudemos construir referências para o papel de professores mediadores (MASETTO, 2000), por exemplo, orientando na construção de dados por meio de objeto de aprendizagem e a partir da interação do aluno com as características do OA.

Para o trabalho pedagógico sobre a sequência de Fibonacci, os alunos também mostraram efetiva participação oriunda tanto pela busca de respostas ao problema inicialmente colocado como pela natureza de interação disponibilizada pelo OA. Isto porque os alunos entenderam que eram responsáveis pelo gerenciamento de informação, pois o OA permitia, por simulação, determinar o ritmo da mudança dos meses do ano e consequentemente também controlar o número de coelhos na tela da animação, respeitando assim os tempos de entendimento de cada aluno.

A formação de grupos necessária para a utilização da simulação digital ocasionou reflexões conjuntas entre os alunos de cada grupo e este ato se intensificou gerando um debate entre todos, no momento em que se buscava encontrar regularidades presentes nos dados das soluções, a fim de sistematizar a sequência numérica.

A maior participação e o envolvimento dos alunos podem ser considerados decorrentes da mudança de ambiente, pois o cotidiano da sala de aula foi modificado e a proposta didática perceptivelmente tornou-se atraente para todos os participantes no processo de ensino e aprendizagem, pois o trabalho envolvia uma situação intrigante para os alunos que poderia ser resolvida também com a utilização do computador e de programas. 
Pudemos novamente construir referências didáticas para a utilização de objetos de aprendizagem, experimentando que podem auxiliar o aluno a formar seus conceitos em um processo participativo e com significado. Quando utilizamos um OA, pudemos entender que nosso papel docente, erroneamente identificado com o de informar ou transferir o conhecimento matemático, e que geralmente acontece em uma aula tradicional, é transformado em função da mediação que podemos realizar entre aluno e situações e informações dispostas pelo uso da informática. Por outro lado, é na natureza da informática que o aluno para aprender deixa de ser um espectador, assumindo também compromisso e responsabilidade por sua aprendizagem proporcionada pela adequada utilização de informática em educação escolar.

A atividade didática era baseada em princípios de uma atividade de investigação matemática, o que nos levou a questionar muito os alunos sobre seus procedimentos e os resultados encontrados. Como o objetivo era oportunizar que os alunos chegassem à Fórmula de Pick, que relaciona a área de uma figura sobre uma malha reticulada, com seus pontos interiores e pontos sobre a figura, as simulações com o OA foram de fundamental importância para que comparações acontecessem e que uma regularidade fosse percebida para a área calculada e os pontos da figura.

O trabalho com a Fórmula de Pick nos levou a situações didáticas nas quais os alunos puderam: reconhecer uma situação problemática, explorar essa a situação, formular questões, organizar dados e formular conjecturas, isto é, levantar hipóteses, além disso, puderam argumentar sobre resultados encontrados e partilhá-los com os outros colegas, ou seja, vivenciamos princípios e momentos de uma atividade de investigação matemática no sentido de Ponte et. al. (1999).

Através do desenvolvimento de ações que compõem uma atividade de investigação é que pudemos perceber o quanto o Geoplano virtual contribuiu para a elaboração dos dados e ações com os mesmos. Sobre as tentativas para encontrar uma fórmula que correspondesse à área de cada figura, o Geoplano também foi fundamental, porque permitiu que todos os alunos participassem ativamente e conjuntamente na atividade proposta. Vimos que os alunos ficaram muito surpresos com a fórmula, pois inicialmente muitos não acreditavam existir uma única fórmula matemática para calcular a área de qualquer polígono. Outros alunos se mostraram motivados com o fato de poderem ter participado da "chegada até à fórmula".

Com este trabalho didático tivemos a oportunidade de também obter parâmetros de como utilizar um objeto de aprendizagem para que este seja considerado um recurso pedagógico que permite alcançar, por exemplo, a sistematização de uma fórmula matemática. Vivenciar uma experiência desta natureza foi uma ótima oportunidade de formação profissional inicial, pois pudemos refletir sobre algumas crenças e mitos acerca da profissão professor. Experiências de sucesso ou insucesso nas atividades desenvolvidas pelos alunos trouxeram a possibilidade de trabalharmos na superação do medo que eles demonstravam em se expressar, e também na compreensão da Matemática. Além disso, pudemos juntamente com os alunos perceber que uma fórmula matemática tem uma história de erros, acertos, partilhas e aceitações, o que certamente leva a ter atitudes muito mais participativas quando se ensina e se aprende Matemática. 


\section{Conclusões}

Descrevemos neste artigo a utilização, por futuros professores de Matemática, de 3 objetos de aprendizagem (OAs) em situações regulares de aula com alunos da Educação Básica. Nossa intenção não era analisar as potencialidades especificas de cada objeto no processo de ensino e aprendizagem, mas sim de, a partir da utilização de OA em contextos regulares de sala de aula, elucidar saberes e implicações para o processo de ensino e aprendizagem tanto para o aluno da Educação Básica como para os futuros professores de Matemática que atuarão neste nível de ensino.

Diante das discussões de cada experiência didática podemos concluir que foi possível elucidar positivas implicações dos OAs em situações didáticas escolares, para alunos e futuros professores. Essas implicações se identificaram com: desenvolvimento de atitudes participativas dos alunos nas aulas, bem como de confiança para prosseguir com procedimentos matemáticos relacionados às atividades, o que perceptivelmente contribuiu para o aprendizado de conceitos matemáticos; entendimento pelos alunos sobre a natureza do conhecimento matemático em termos de resultado da atividade humana diante de uma situação problema que se busca resolver. Nessas implicações pudemos entender a contribuição da utilização de informática para a constituição de saberes conceituais, procedimentais e atitudinais. Especificamente aos futuros professores, as experiências didáticas com OAs constituíram-se fontes de elaboração e reelaboração de saberes docentes associados a saberes específicos do conteúdo matemático, como também à gestão didática dos mesmos, incluindo a vivência e a reflexão sobre o conceito de professor mediador.

\section{Referências}

Área de Figuras Geométricas em Malha Pontilhada (2011). Disponível em: http://portaldoprofessor.mec.gov.br/fichaTecnicaAula.html?aula=30282. Acesso em $\mathrm{Jul} / 2012$.

Audino, D. F; Nascimento, R. F. (2010). Objetos de aprendizagem- diálogos entre conceitos e uma nova proposição aplicada à educação. In: Revista Contemporânea de Educação, vol. 5, n. 10, p. 128-148.

Brasil. (1998). Secretaria de Educação Fundamental. Parâmetros curriculares nacionais: Matemática/ Secretaria de Educação Fundamental. - Brasília: $\mathrm{MEC} / \mathrm{SEF}, 148 \mathrm{p}$.

Brasil. (2008). Ministério da Educação. Banco Internacional de Objetos Educacionais (BIOE).Disponível em: http://objetoseducacionais2.mec.gov.br/. Acesso em Ago/2014.

Colômbia. (2004). Ministerio da Educación Nacional. Colombia Aprende- La Red del Conocimiento. Disponível

em: http://www.colombiaaprende.edu.co/html/home/1592/w3-channel.html. Acesso em Ago/2014.

Direne A. I et. al. (2009). Objetos de Aprendizagem Generalizáveis para o Currículo de Matemática do Ensino Médio. In: WIE - XV Workshop sobre Informática na Escola. 
Sociedade Brasileira de Computação, Bento Gonçalves/RS. Disponível em: http://www.br-ie.org/index.php/anaiswie. Acesso em: Mai/2014.

Fabri, L. et. al. (2012). Repositório institucional de objetos de aprendizagem utilizando DSpace. In: Anais do $23^{\circ}$ Simpósio Brasileiro de Informática na Educação.Disponível em: http://www.br-ie.org/index.php/anaissbie. Acesso em: $\mathrm{Mai} / 2014$.

International Society for Technology in Education. (2009). Disponível em http://www.iste.org/. Acesso em: Jun/2009.

Kenski, V. M. (2007). Tecnologias também servem para fazer educação In: Educação e Tecnologias O Novo Ritmo da Informação. 3.ed. Campinas, SP: Papirus, p.43-62.

Leite, M. A.; Castro Filho, J. A. (2006). Aprendizagem de conceitos matemáticos e interação entre pares durante o uso de um objeto de aprendizagem. In: XII Workshop de Informática na Escola, Proceedings of XXVI Congresso da SBC, Campo Grande.

Ludoteca do Instituto de Física da USP. O Problema dos coelhos - Leonardo de Pisa Disponível em http://www.ludoteca.if.usp.br/ripe/index.php. Acesso em: Mar/2012.

Masetto, M. T. (2000). Mediação pedagógica e o uso da tecnologia. In: Novas Tecnologias e Mediação Pedagógica, Moran, J. M. et. al. Campinas/SP, Papirus.

Nascimento, A. C. A. (2007). Objetos de Aprendizagem: a distância entre a promessa e a realidade. In: Objetos de aprendizagem: uma proposta de recurso pedagógico. (Org): Prata, C. L.; Nascimento, A. C. A., Brasília: MEC, SEED, p.135-145.

Ponte, J. et. al. A relação professor-aluno na realização de investigações matemáticas. Lisboa: Projecto MPT e APM, 1999.

Prata, C. L.; Nascimento, A. C. A. A. (2007). Objetos de aprendizagem: uma proposta de recurso pedagógico. Brasília: MEC, SEED.

Tarouco, L. M. R. et. al. (2008). Reusabilidade de objetos educacionais. Disponível em: http://www.cinted.ufrgs.br/renote/fev2003/artigos/marie_reusabilidade.pdf Acesso em: Ago/2008.

Valente, J. A. (1999). Informática na educação no Brasil: Análise e contextualização histórica. In: O Computador na Sociedade do Conhecimento, Valente, J. A., Campinas: UNICAMP/NIED, p. 1-4.

Valente, J.A. (2002). A espiral da aprendizagem e as tecnologias da informação e comunicação: repensando conceitos. In: JOLY, M. C. A Tecnologia no Ensino: implicações para a aprendizagem. São Paulo: Casa do Psicólogo, p. 15-37.

Macedo, L. et.al. (2008). Análise do Uso de um Objeto de Aprendizagem Digital no Ensino de Álgebra - versão final. In: Anais do XIX Simpósio Brasileiro de Informática na Educação. http://www.br-ie.org/index.php/anaissbie. Acesso em: Jun/2014. 